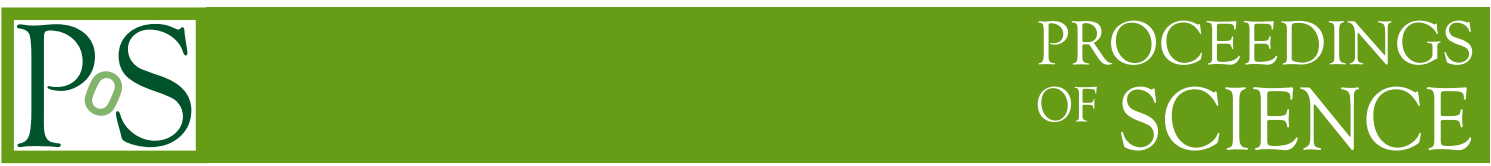

\title{
Polarised Drell-Yan measurements at COMPASS-II
}

\author{
Michela CHIOSSO*† \\ ( INFN, Sezione di Torino and University of Torino) \\ E-mail: chiosso@to.infn.it
}

\begin{abstract}
Much of the information that exists today about Transverse Momentum Dependent Parton Distribution Functions (TMDs) comes from SIDIS measurements with unpolarised and polarised beams and targets where they appear convoluted with fragmentation functions (FFs). Drell-Yan (DY) measurements are complementary to those by SIDIS experiments, as they allow to measure convolutions of only Parton Distribution Functions (PDFs) without involving FFs. Moreover, given the T-odd character of both Sivers and Boer-Mulders functions, the sign of these TMDs is expected to be reversed when observed from SIDIS or from DY. Measurements of SIDIS were performed by Compass in the period 2002 to 2007 and in 2010, using a naturally polarised $\mu^{+}$ beam and a solid state target polarised either longitudinally or transversely with respect to the beam direction. Now the COMPASS-II Experiment has the unique opportunity to access TMDs from single-polarised Drell-Yan processes as well, allowing to test the sign change prediction for the first time. We present here the future polarised DY measurements at COMPASS-II, which are foreseen to start in late 2014.
\end{abstract}

XXI International Workshop on Deep-Inelastic Scattering and Related Subject -DIS2013, 22-26 April 2013

Marseilles,France

*Speaker.

${ }^{\dagger}$ on Behalf of the COMPAss Collaboration. 


\section{The Drell-Yan Programme}

The main goal of COMPASS-II DY programme is to measure the process $\pi^{-} p^{\uparrow} \rightarrow \mu^{+} \mu^{-} X$ with a $190 \mathrm{GeV} \pi^{-}$beam scattered off a transversely polarised proton target $\left(\mathrm{NH}_{3}\right)$.

By performing the measurements of target spin (in)dependent asymmetries in DY reaction and comparing the results with ones obtained in SIDIS, we will be able for the first time ever to verify the universality of TMD approach for the description of these reactions. This would be a crucial test of QCD in the non-perturbative regime. In Fig. 1 the $Q^{2}$ versus $x$ phase-space region expected

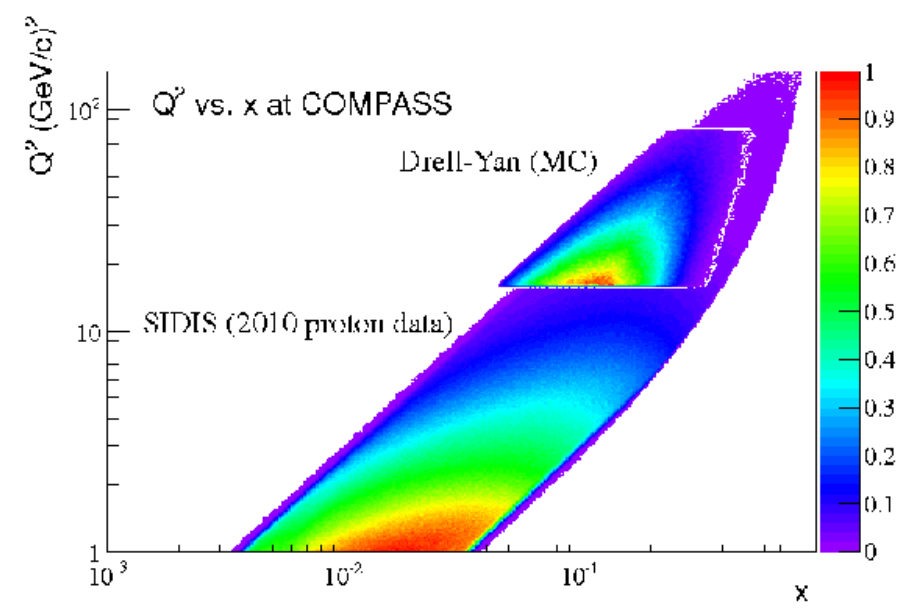

Figure 1: $Q^{2}$ versus $x$ distribution, from Drell-Yan Monte-Carlo events with $4 \leq M_{\mu \mu} \leq 9 \mathrm{GeV} / \mathrm{c}^{2}$ and from 2010 SIDIS data with transversely polarized $\mathrm{NH}_{3}$ target.

to be covered by the COMPASS DY measurements is superimposed with the one accessed by the SIDIS processes at COMPASS with transversely polarized $\mathrm{NH}_{3}$ s target. So we expect good overlap of the phase-space regions for DY and SIDIS. $Q^{2}$ in Fig. 1 is the dimuon invariant mass squared, and $x$ stands for the momentum fraction of the annihilated quark(antiquark) from the target nucleon.

By doing single transversely polarised DY measurements we can access four azimuthal asymmetries, what gives us an input on different convolutions of PDFs and TMDs:

- $A_{U}^{\cos 2 \phi}$ gives access to the Boer-Mulders functions, $h_{1}^{\perp}$, of incoming hadrons,

- $A_{T}^{\sin \phi_{S}}$ to the Sivers function, $f_{1 T}^{\perp}$, of the target nucleon,

- $A_{T}^{\sin \left(2 \phi+\phi_{S}\right)}$ to the Boer-Mulders functions of beam hadron and to the pretzelosity function, $h_{1 T}^{\perp}$, of the target nucleon,

- $A_{T}^{\sin \left(2 \phi-\phi_{S}\right)}$ to Boer-Mulders functions of beam hadron and to the transversity function, $h_{1}$, of the target nucleon.

To disentangle the PDFs in each of these asymmetries requires certain knowledge of other PDFs. The simplest case is the extraction of the Sivers function, which is convoluted with the well known pion unpolarised PDF. More complex is to access the transversity and pretzelosity of the nucleon for which the knowledge of the Boer-Mulders function of the pion is needed. There are two different scenarios to evaluate this function: 
1. measure the $A_{T}^{\sin \left(2 \phi-\phi_{S}\right)}$ asymmetry and use the parametrization of $h_{1}$ from Refs. [2, 3] to extract Boer-Mulders function of the pion;

2. use the already existing measurements of $A_{U}^{\cos 2 \phi}$ and the information on the Boer-Mulders function of the target nucleon. This asymmetry was measured in the unpolarized $\pi+N$ Drell-Yan process by the NA10 and E615 Collaborations $[4,5,6]$ and more recently by the E866/NuSea Collaboration at FNAL [7, 8] in $p+N$ Drell-Yan reaction.

Another topic which will be investigated is the so-called J/ $\psi$-DY duality [9]. In spite of the large amount of experimental data on $\mathrm{J} / \psi$ production in various reactions, the mechanism is still unclear. Since the $\mathrm{J} / \psi$ and the $\gamma$ are both vector particles and the helicity structure of $\bar{q} q(J \psi)$ and $(\bar{q} q) \gamma^{*}$ couplings is the same, it is believed that an analogy in the production mechanism might occur at low energies where the $\bar{q} q$ annihilation dominates. The study of the $\mathrm{J} / \psi$ production in the dilepton decays channel will allow us to check the duality hipothesis. Moreover such a possibility to use $\mathrm{J} / \psi$ production for the extraction of PDFs is very attractive because the dilepton production rate in the $\mathrm{J} / \psi$ production region is factor of 30 higher than in the continuum region above the $\mathrm{J} / \psi$ mass.

\section{Acceptance, event rate and statistical precision}

A full Monte-Carlo simulation was performed to evaluate the COMPASS spectrometer acceptance in the invariant mass intervals $4 \leq M_{\mu \mu} \leq 9 \mathrm{GeV} / \mathrm{c}^{2}$ and $2 \leq M_{\mu \mu} \leq 2.5 \mathrm{GeV} / \mathrm{c}^{2}$. These two regions are considered to be the best mass intervals for Drell-Yan analysis, avoiding the large combinatorial background that shall dominate at lower dimuon masses, and excluding the $\phi, J / \psi$ and $\Upsilon$ vector-meson resonances. The first (high) mass region certainly provides a cleaner sample of DY events, because of the very small contribution from uncorrelated combinatorial muon background (originating from pion and kaon decays) and open-charm semileptonic decays (i.e $D \bar{D}$ decays into muons). It is particularly interesting, as it covers mostly the region of valence quarks, but the DY cross-section for such masses is almost a factor 5 smaller than in the second (intermediate) mass region. The simulation results [1] show that the COMPASS spectrometer acceptance is $35 \%$ in the high mass region and $43 \%$ in the intermediate mass region.

In the proposed Drell-Yan measurement with a $190 \mathrm{GeV} \pi^{-}$beam and a beam intensity of $6 \mathrm{x}$ $10^{7}$ particles/s we can achieve a luminosity of $1.2 \times 10^{32} \mathrm{~cm}^{-2} \mathrm{~s}^{-1}$. The DY useful event rates will amount to 800 events/day for the high mass region and 4900 events/day for the intermediate mass region. In Fig. 2 the expected statistical error of the Sivers asymmetry, assuming one year of data taking, together with the theoretical prediction from Anselmino et al. is shown. Assuming two years (280 days) of data taking, the expected statistical errors in the azimuthal asymmetries are as given in table 1. In Fig. 3 projections of the expected statistical errors of the Sivers and BoerMulders asymmetries, assuming two years of data taking, together with the theory predictions [11], are shown.

As the measurement is statistics limited, the luminosity optimisation is mandatory. The installation of an hadron absorber downstream of the target will reduce the high secondary particle flux produced by the interaction of the pion beam in the target and, consequently, the tracking detector occupancies. This will make an increase in the intensity of the incident pion beam possible. The absorber, $236 \mathrm{~cm}$ long, is made by alumina $\left(\mathrm{Al}_{2} \mathrm{O}_{3}\right)$, with a $140 \mathrm{~cm}$ long tungsten beam plug inside 


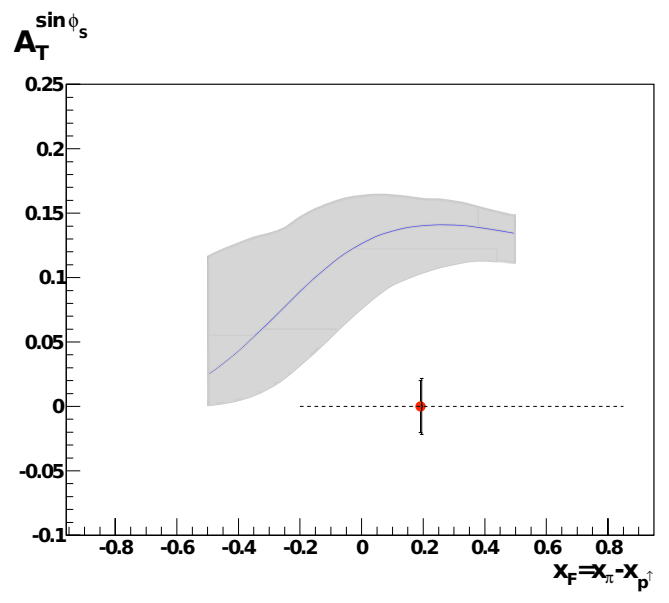

Figure 2: Expected statistical error of the Sivers asymmetry in the dimuon mass range $4 . \leq M_{\mu \mu} \leq 9$. $\mathrm{GeV} / \mathrm{c}^{2}$, assuming one year of data taking (140 days). The smaller error bar denotes the statistical error, while the larger corresponds to the quadratic sum of statistical and systematic error. The theoretical prediction of the asymmetry from Anselmino et al. is also shown.

\begin{tabular}{|c|c|c|c|}
\hline Dimuon mass $\left(\mathrm{GeV} / \mathrm{c}^{2}\right)$ & $2<M_{\mu \mu}<2.5$ & $\mathrm{~J} / \psi$ region & $4<M_{\mu \mu}<9$ \\
\hline$\delta A_{U}^{\cos 2 \phi}$ & 0.0020 & 0.0013 & 0.0045 \\
\hline$\delta A_{T}^{\sin \phi_{S}}$ & 0.0062 & 0.0040 & 0.0142 \\
\hline$\delta A_{T}^{\sin \left(2 \phi+\phi_{S}\right)}$ & 0.0123 & 0.008 & 0.0285 \\
\hline$\delta A_{T}^{\sin \left(2 \phi-\phi_{S}\right)}$ & 0.0123 & 0.008 & 0.0285 \\
\hline
\end{tabular}

Table 1: Expected statistical errors in the asymmetries, assuming 2 years of data-taking, beam momentum of $190 \mathrm{GeV} / \mathrm{c}$.

it to stop the non-interacted beam. The alumina absorber will stop the secondary particles flux, minimising multiple scattering; this is essential to disentangle the two oppositely polarised target cells in the track reconstruction.

A scintillating fibers vertex detector will be placed between the target and the absorber, in order to further improve the resolution in the vertex position reconstruction and, consequently, the two target cells separation.

\section{Feasibility of the measurement}

In order to study the feasibility of the proposed polarised Drell-Yan programme and to evaluate in a realistic way the achieavable statistical accuracies, short beam tests were performed in 2007, 2008, 2009 and 2012. During the last one a prototype hadron absorber, made of concrete and stainless steel, 1 meter long each, and with a tungsten beam plug in its center, was installed downstream of the target. A $\pi^{-}$beam with momentum $190 \mathrm{GeV} / \mathrm{c}$ was used. The beam intensity was $8 \times 10^{7}$ pions/spill (spill length of 9.6s), except for two runs when it was eventually increased to $1.5 \times 10^{8}$ pions/spill. The obtained dimuon mass spectrum, shown in Fig. 4, is in agreement 

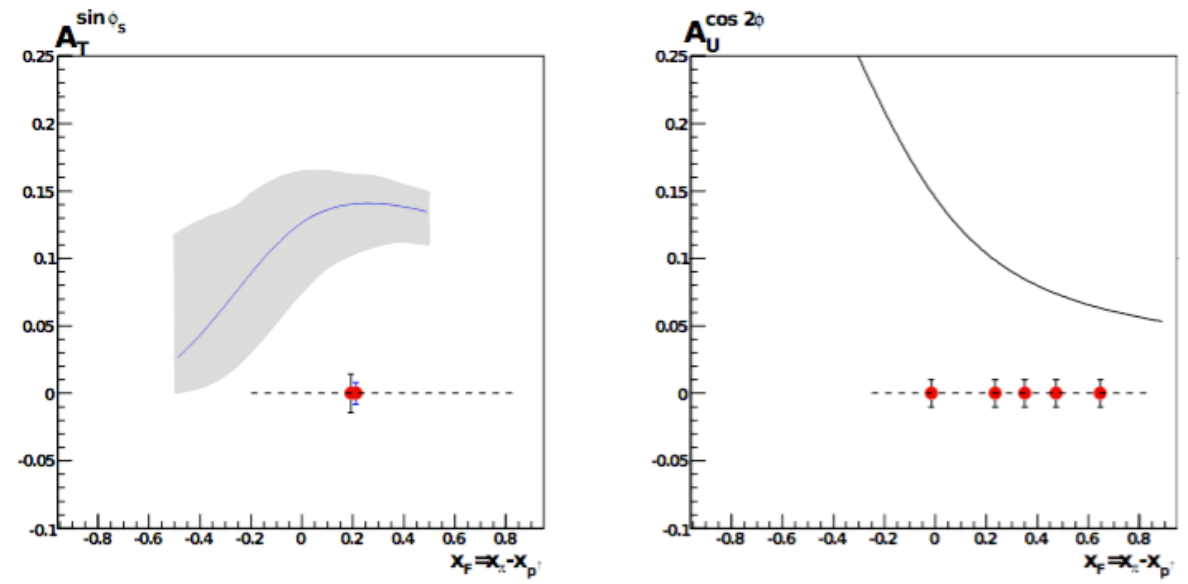

Figure 3: Expected statistical errors and theoretical predictions [11] on the Sivers (left) and Boer-Mulders (right) asymmetries for a DY measurement in the dimuon mass range $4 . \leq M_{\mu \mu} \leq 9$. GeV/ $\mathrm{c}^{2}$. The smaller error bar in Sivers denotes the statistical error, while the larger corresponds to the quadratic sum of statistical and systematic error.

with the Monte-Carlo simulations. The number of found $\mathrm{J} / \psi, 3170 \pm 70$, agrees well with the expectation of $3600 \pm 600$ from the Monte-Carlo. The number of DY events $(84 \pm 10)$ found in the high mass ragion is also in good agreement with the expected number of $110 \pm 22$.

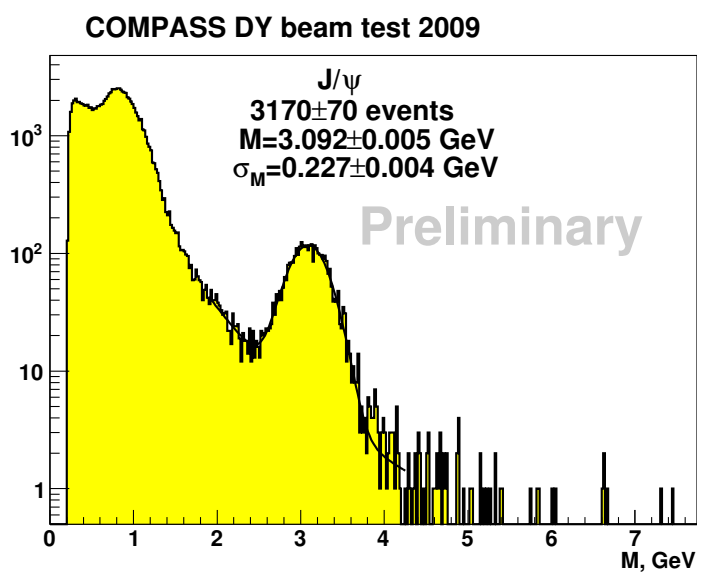

Figure 4: The measured $\mu^{+} \mu^{-}$invariant mass distribution. The number of events is obtained from the fit in the $\mathrm{J} / \psi$ region.

\section{Conclusions}

The COMPASS-II DY programme is part of the COMPASs-II Proposal [1], approved in December 2010 by the CERN Research Board. We already entered this second phase of the COMPASS experiment in 2012 with a pion/kaon polarisability measurement through Primakoff reaction and a pilot run for generalised parton distribution functions measurements (GPDs) through deeply virtual Compton scattering (DVCS) and deeply virtual meson production (DVMP). 
The Drell-Yan programme will start at the end of 2014, when CERN accelerator shutdown will be over and will continue over the whole 2015. We expect to have first physics results for polarised DY in the beginning of 2016.

\section{References}

[1] COMPASS Collaboration “COMPASS-II proposal” CERN-SPSC-2010-014 SPSC-P-340 17 May 2010

[2] M. Anselmino et al. Phys. Rev. D75 2007054032

[3] M. Anselmino et al. Nucl. Phys. Proc. Suppl. 191200998

[4] NA10, S. Falciano et al. Z. Phys. C31 1986513

[5] J. S. Conway et al. Phys. Rev. D39 193992

[6] NA10, S. Guanziroli et al. Z. Phys.C37 1988545

[7] FNAL-E866/NuSea, L. Zhu et al. Phys. Rev. Lett. 99

[8] FNAL-E866/NuSea, L. Zhu et al. Phys. Rev. Lett. 102

[9] Collins J. C. Phys. Lett. B 2002 V.536 43

[10] Anselmino M. et al. Proc. Workshop on Transverse Polarization Phenomena in Hard Scattering Processes ed Ciullo G et al. (Ferrara: World Scientific) 2009138

[11] M. Anselmino et al. Phys. Lett. B594 200497 